\title{
Anti-SAE1 Antibodies and Dermatomyositis: A Case Series of Three Patients
}

\author{
Claire Wilson $\mathrm{BS}^{1^{*}}$, Brandon Roman $\mathrm{BS}^{1^{*}}$, Kurt A. Ashack MD${ }^{1}$, Iris K. Aronson MD \\ ${ }^{1}$ Department of Dermatology, University of Illinois at Chicago College of Medicine, Chicago, IL \\ ${ }^{*}$ Co-first authors
}

\section{ABSTRACT}

To date, few patients with anti-SAE1 dermatomyositis (DM) have been described, and usually in large descriptive cohort studies including all DM subtypes. Thus, it is increasingly important to further describe this rare subtype to solidify cutaneous findings, associated symptoms, and potential therapeutic options. Herein, this case series describes three patients with anti-SAE1 DM with respect to their clinical and laboratory findings, and also their response to treatment. All three patients eventually presented with classic cutaneous manifestations of dermatomyositis, however, Case 2 presented initially with cutaneous manifestations not described in the literature. Furthermore, two patients presented with dysphagia, with Case 2 experiencing a life-threatening dysphagia not typically described in the literature. Two of three patients also presented with early signs of interstitial lung disease. Lastly, cutaneous disease was only responsive to immunosuppressants in one patient. Unfortunately, this observational case-series consisted of only three patients, limiting the generalizability of our results. However, this study provides further support of the recalcitrant nature of this DM subtype, and commonly affected extracutaneous systems including the gastrointestinal and pulmonary systems. Larger studies of this patient population are needed to further confirm our associated findings.

\section{INTRODUCTION}

Diagnosing clinically amyopathic dermatomyositis (CADM) is a challenge for clinicians due to the lack of classic muscle findings associated with dermatomyositis (DM) and the wide array of clinical presentations depending on the associated antibody subtype. ${ }^{1}$ In 2007, Betteridge et al. described a new subtype of CADM associated with Anti-small ubiquitin-like modifier activating enzyme 1(anti-SAE1) antibodies. ${ }^{2}$ This subtype of CADM is often recalcitrant to treatment and may be associated with severe cutaneous disease, dysphagia, and interstitial lung disease. ${ }^{3}$ To date, few patients with anti-SAE1 DM have been described, and usually in large descriptive cohort studies including all DM subtypes. Thus, it is increasingly important to further describe this rare subtype to solidify cutaneous findings, associated symptoms, and potential therapeutic options. Herein, we describe three patients with anti-SAE1 DM, with one patient presenting with a previously undescribed cutaneous manifestation.

\section{CASE PRESENTATION}

\section{Case 1}

A 56-year-old Caucasian woman presented with two months of an erythematous and pruritic eruption on the scalp, face, neck,

November 2020 Volume 4 Issue 6 


\section{SKIN}

Figure 1. Case 1 Clinical Findings. Cutaneous examination of Case 1 revealed $(\mathbf{A})$ confluent erythematous patches involving the upper arms bilaterally, (B) the upper chest, (C) and the upper back. The patient also had erythematous and edematous plaques involving the bilateral upper eyelids, and erythematous macules over the dorsal hands.

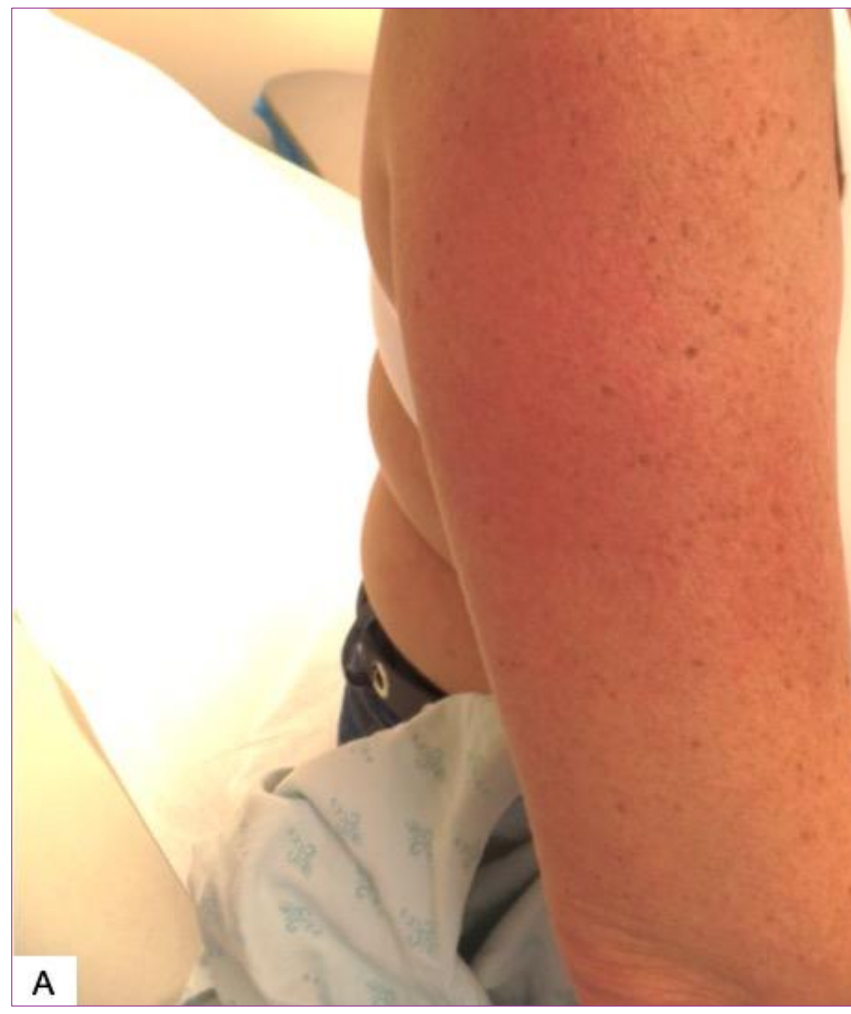

trunk, and upper extremities (Figure 1).At presentation, patient did not report any myalgias, weakness, dysphagia, dyspnea, or weight loss. Over the next several months, patient developed muscle weakness and dysphagia, which responded to a combination of monthly intravenous immune globulin (IVIg), mycophenolate mofetil (MMF), and prednisone. However, her cutaneous disease did not improve with the above treatments in addition or to intramuscular and topical corticosteroids and worsened with hydroxychloroquine, which was then discontinued.

\section{Case 2}

A 57-year-old African American woman presented with a 1.5 month history of lesions clinically consistent with discoid lupus erythematosus (DLE) (Figure 2). The patient
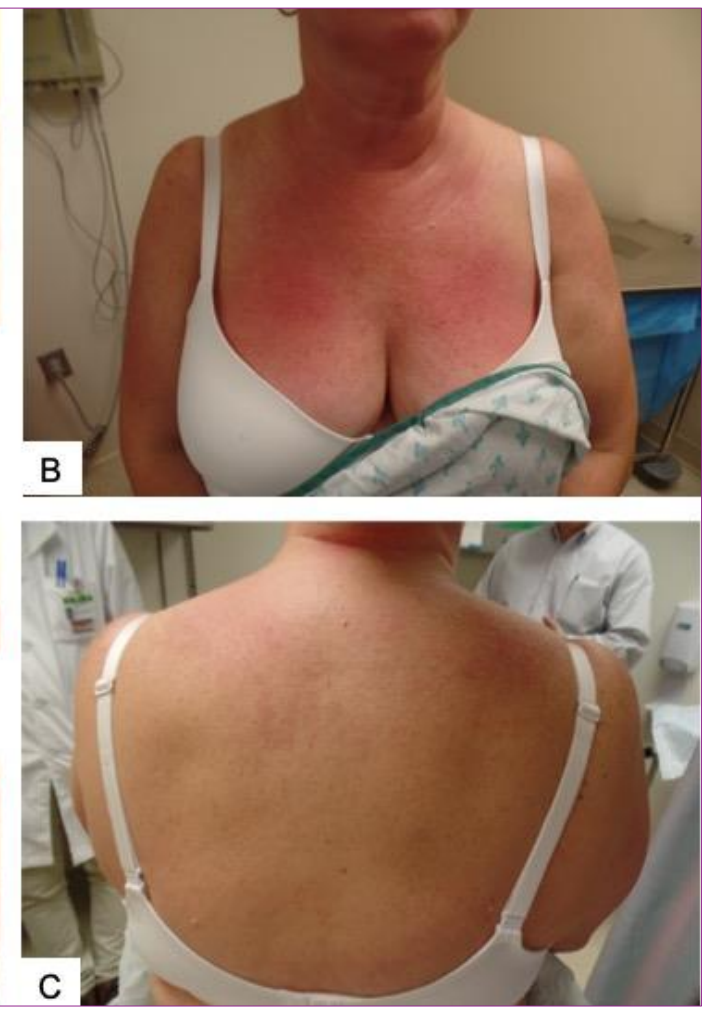

denied myalgias, weakness, or dyspnea. Hydroxychloroquine resolved her eroded DLE-like lesions, with multiple areas of scarring left behind.

Three months later, she developed extensive violaceous, erythematous, and edematous macules, papules, patches, and plaques on the face, neck, chest, arms, dorsal hands, and lateral thighs consistent with a diagnosis of dermatomyositis. She was subsequently hospitalized due to severe dysphagia, which improved with intravenous methylprednisolone. Methotrexate, oral corticosteroids, and topical corticosteroids were started in addition to hydroxychloroquine with no significant improvement in cutaneous manifestations. 


\section{SKIN}

Figure 2. Case 2 Clinical Findings. Clinical exam of Case 2 revealed $(\mathbf{A})$ diffuse erythematous, jagged and eroded plaques of the trunk and upper and lower extremities bilaterally. The patient also had (B) erythematous and eroded plaques at different stages affecting the upper anterior legs and lateral thighs, and $(\mathbf{C})$ erythematous and erosive papules and plaques overlying the dorsal hands.
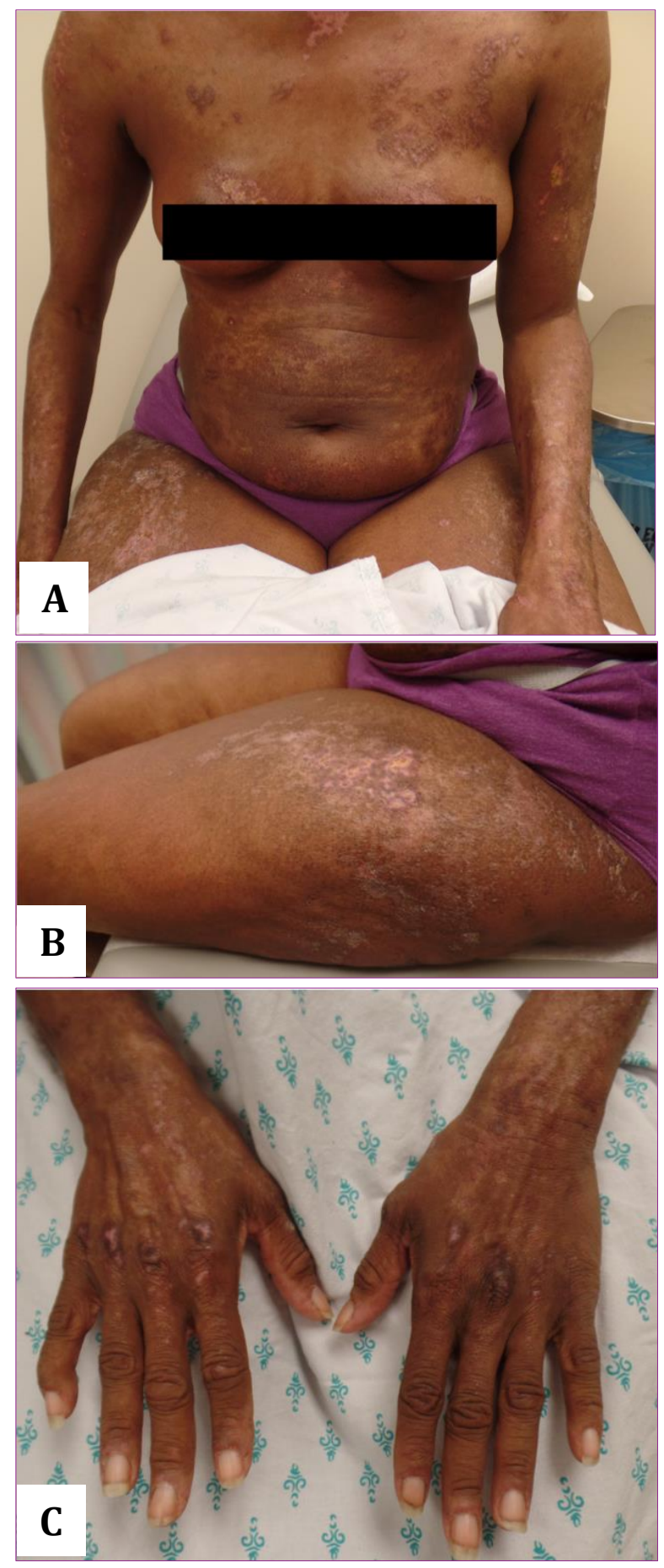

Case 3

A 66-year-old woman presented with over a one-year history of a diffuse pruritic, burning erythematous eruption covering the face, trunk, upper extremities, and knees (Figure 3). On presentation, patient reported no myalgias, dysphagia, dyspnea, or weight loss. However, over time, the patient developed generalized weakness, which improved (along with cutaneous findings) with topical and oral corticosteroids and MMF.

Histopathology for all three cases demonstrated a vacuolar interface dermatitis with stromal mucin deposition and nonspecific direct immunofluorescence findings. Additionally, a dermatomyositis panel was positive for anti-SAE1 antibodies for all three patients. Table 1 further describes clinical and laboratory findings in our patients.

\section{DISCUSSION}

Similar to the majority of patients with SAE antibody DM, our patients presented with skin disease prior to the development of other symptoms. Presentation with dysphagia occurs less frequently, varying between $29 \%$ to $78 \%$ of initial presentations. $^{3-5}$ The degree of dysphagia varies as well, but can be severe and life threatening as demonstrated by Case 2 .

Myositis typically develops several months after the onset of skin findings in this specific patient population, with elevated creatinine kinase (CK) levels being very common. ${ }^{3}$ Elevated CK levels among these patients have been noted by Peterson et al. (2/17 patients), Muro et al. (7/7 patients), and Ge et al. (6/11 patients). ${ }^{3-5}$ Among the Western cohorts studied by $\mathrm{Ge}$ et al., the Betteridge study found $2 / 2$ and 9/11 patients with elevated CK, and the Tarricone study found 
Figure 3. Case 3 Clinical Findings. Dermatological examination of Case 3 revealed (A) erythematous patches covering the forehead, periocular area, cheeks, chest and arms, abdomen, and (B) back. There were also (C) erythematous papules on the dorsal hands in addition to ragged cuticles. (D) Both knees also had erythematous to violaceous patches bilaterally.
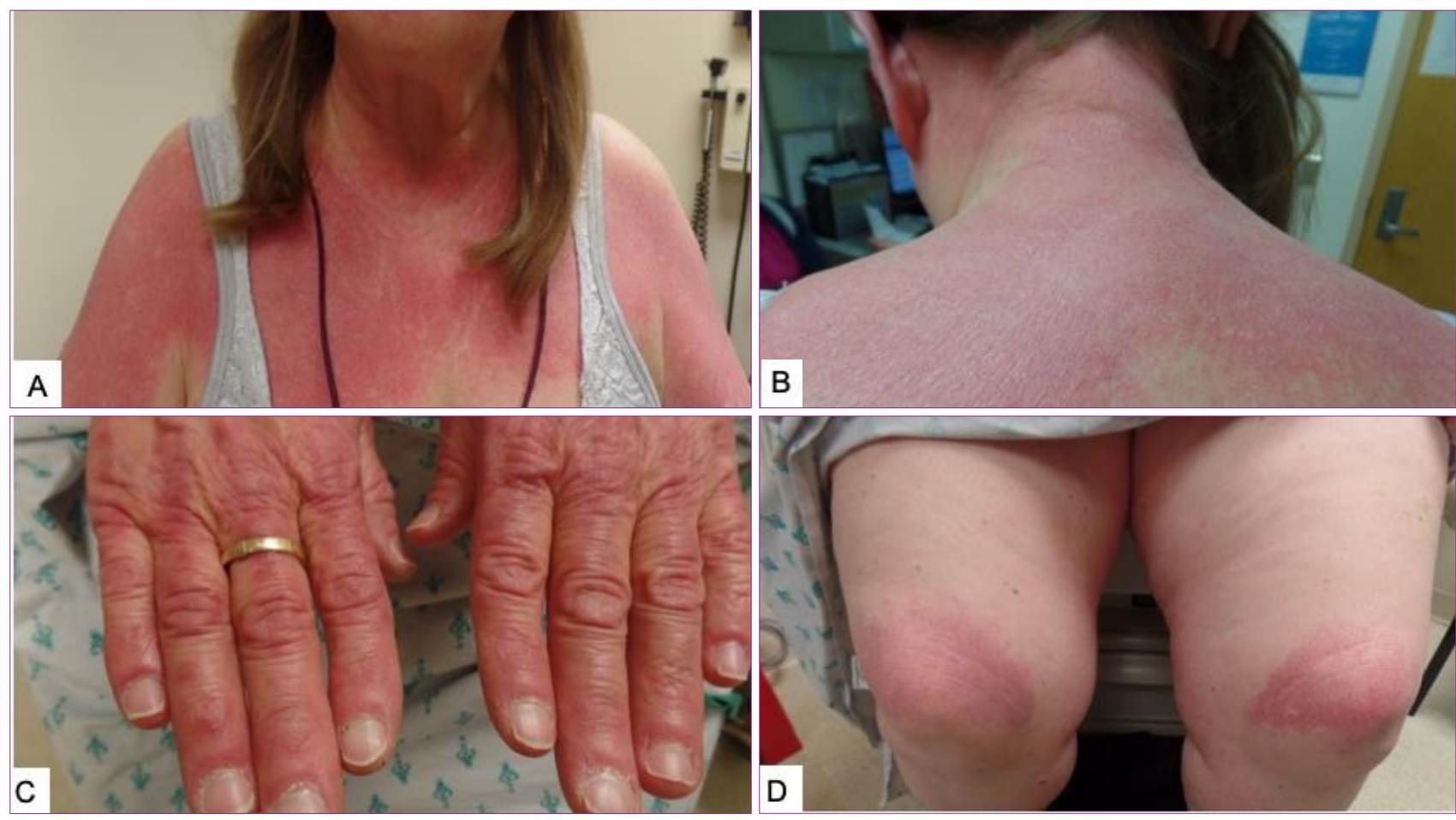

4/5 patients with elevated CK. Among the Asian cohorts, the Fujimoto study found 5/7 patients with elevated CK. ${ }^{3}$ Within our small cohort, CK and aldolase levels were initially all within normal limits, however, CK levels became elevated in Cases 1 and 2. Case 3 did develop severe subjective weakness despite normal CK. Furthermore, two of our three patients were ANA positive, with one patient demonstrating anti-histone antibodies, and another with anti-ssDNA antibodies, findings not described in the literature with respect to this specific subset of DM patients.

Two of the three patients developed CT findings consistent with interstitial lung disease, another commonly reported finding among this patient population. This report supports this complicating feature in antiSAE1 patients. To date, none of our patients have been diagnosed with a malignancy, which aligns with other studies. ${ }^{3}$

Of our three patients, Case 2 deviated the most from all reported cases with respect to cutaneous findings. Though ulcerations have been reported in dermatomyositis, a case of diffuse eroded plaques has not been reported in the onset of DM in the literature. Though her initial cutaneous ulcerations improved with hydroxychloroquine and prednisone, the diffuse poikilodermatous erythroderma that later developed did not. Rituximab infusions have also not been beneficial for Case 2.

Furthermore, Case 3 showed very good response to the addition of MMF with oral steroids, with remarkable improvement in her cutaneous disease and muscular weakness. Case 1 showed improvement in 
Table 1: Summary of Patient Characteristics

\begin{tabular}{|c|c|c|c|}
\hline & \multicolumn{3}{|c|}{ Patient \# } \\
\hline & 1 & 2 & 3 \\
\hline Age & 56 & 57 & 66 \\
\hline Gender & $\mathrm{F}$ & $\mathrm{F}$ & $\mathrm{F}$ \\
\hline Dysphagia & + & + & - \\
\hline $\begin{array}{l}\text { Muscular } \\
\text { Weakness }\end{array}$ & $+^{a}$ & - & $t^{a}$ \\
\hline Holster Sign & - & + & + \\
\hline $\begin{array}{l}\text { Heliotrope } \\
\text { Sign }\end{array}$ & + & + & + \\
\hline V sign & + & - & + \\
\hline $\begin{array}{l}\text { Gottron's } \\
\text { Papules }\end{array}$ & - & + & + \\
\hline Shawl Sign & + & - & + \\
\hline $\begin{array}{l}\text { Skin } \\
\text { Ulceration }\end{array}$ & - & + & - \\
\hline Myalgia & - & - & - \\
\hline Arthralgia & - & - & - \\
\hline $\begin{array}{l}\text { Pulmonary } \\
\text { Findings }\end{array}$ & $+^{\mathrm{b}}$ & $+^{\mathrm{C}}$ & - \\
\hline Cancer & - & - & - \\
\hline $\begin{array}{l}\text { Family } \\
\text { History of } \\
\text { Cancer }\end{array}$ & $++^{d}$ & $+^{e}$ & $++^{f}$ \\
\hline CK/aldolase ${ }^{g}$ & Normal & Normal & Normal \\
\hline ESR & Elevated & Elevated & Normal \\
\hline CRP & Normal & Normal & Normal \\
\hline ANA & $+^{h}$ & - & $t^{i}$ \\
\hline Anti-histone & + & $\mathrm{N} / \mathrm{A}$ & $\mathrm{N} / \mathrm{A}$ \\
\hline Anti-SS DNA & - & - & + \\
\hline HCQ Use & $+^{\mathrm{j}}$ & + & - \\
\hline
\end{tabular}

ANA - anti-nuclear antibody; HCQ - hydroxychloroquine

a Objective clinical weakness develops in Cases 1 and 3 after having cutaneous manifestations alone for at least six months.

${ }^{\mathrm{b}}$ A chest CT with contrast showed bibasilar wedge-shaped consolidations suggesting interstitial lung disease (ILD).

${ }^{c}$ CT revealed tree in bud opacities in the left lower lung representing small airway disease.

${ }^{d}$ Family history for case 1 included two sisters with breast cancer.

e Family history for case 2 included breast cancer in one family member.

f Family history for case 3 included a mother with sarcoidosis and uterine cancer, and a cousin with breast cancer.

9 Initial CK and aldolase levels were all normal

${ }^{\text {h }}$ ANA titer 1:640, speckled pattern.

'ANA titer 1:320, homogeneous pattern.

j Hydroxychloroquine (HCQ) was discontinued due to cutaneous adverse reactions. her muscular weakness with IVIg, but her cutaneous disease remains recalcitrant. Overall, this small group of patients shows variable cutaneous response to treatment.

Finally, a majority of DM cases are considered idiopathic, but cases of medication induced dermatomyositis have been reported. ${ }^{6}$ It is important to note that two of our three patients were on statin medications prior to the development of skin disease. Unfortunately, their disease persisted despite discontinuation of this medication, and statin involvement should be considered in each case of DM.

Limitations of this study include our small sample size of three patients. This limits the generalizability of our results. Therefore, it is important that more case-series limited to this specific subtype be published to help identify clinical features and possible treatments.

\section{CONCLUSION}

Herein, we add three patients to the literature with this uncommon subtype of CADM. Additionally, we demonstrate several key clinical and laboratory findings that deviate from the average phenotype of CADM patients with anti-SAE1 antibodies.

Conflict of Interest Disclosures: None

Funding: None

Corresponding Author:

Kurt A. Ashack, MD

Dermatology Associates of West Michigan

1740 East Paris Ave. SE

Grand Rapids, MI 49546

Phone: 616-949-5600

Fax: 616-949-6571

Email: kurt.ashack@gmail.com 


\section{References:}

1. Bailey EE, Fiorentino DF. Amyopathic dermatomyositis: definitions, diagnosis, and management. Curr Rheumatol Rep 2014;16:465.

2. Bodoki L, Nagy-Vincze M, Griger Z, Betteridge Z, Szollosi L , Danko K. Four dermatomyositisspecific autoantibodies-anti-TIF1gamma, antiNXP2, anti-SAE and anti-MDA5-in adult and juvenile patients with idiopathic inflammatory myopathies in a Hungarian cohort. Autoimmun Rev 2014;13:1211-9.

3. Ge Y, Lu X, Shu X, Peng Q, Wang G. Clinical characteristics of anti-SAE antibodies in Chinese patients with dermatomyositis in comparison with different patient cohorts. Sci Rep 2017;7:188.

4. Muro Y, Sugiura K, Akiyama M. Low prevalence of anti-small ubiquitin-like modifier activating enzyme antibodies in dermatomyositis patients. Autoimmunity 2013;46:279-84.

5. Peterson LK, Jaskowski TD, La'ulu SL, Tebo AE. Antibodies to small ubiquitin-like modifier activating enzyme are associated with a diagnosis of dermatomyositis: results from an unselected cohort. Immunol Res 2018;66:431-6.

6. Borges IBP, Silva MG, Misse RG, Shinjo SK. Lipid-lowering agent-triggered dermatomyositis and polymyositis: a case series and literature review. Rheumatol Int 2018;38:293-301. 